\title{
LA INCLUSIÓN SOCIAL Y LABORAL DE LAS PERSONAS CON DISCAPACIDAD INTELECTUAL MEDIANTE LOS PROGRAMAS DE EMPLEO CON APOYO. UN RETO PARA LA ORIENTACIÓN
}

\author{
SOCIAL AND LABOUR INCLUSION OF PEOPLE WITH INTELLECTUAL \\ DISABILITY THROUGH SUPPORTED EMPLOYMENT PROGRAMS. \\ A CHALLENGE FOR GUIDANCE
}

\author{
Inmaculada Egido Gálvez* \\ Universidad Complutense de Madrid \\ Rosario Cerrillo Martín**, Asunción Camina Durantes*** \\ Universidad Autónoma de Madrid
}

\begin{abstract}
RESUMEN
El empleo con apoyo constituye actualmente una de las estrategias más prometedoras para la inserción laboral de las personas con discapacidad. Este artículo sintetiza un proyecto de investigación en el que se ha implementado y evaluado un Programa de formación para la inserción laboral de jóvenes con discapacidad intelectual desarrollado en el ámbito universitario. Se describen los principales rasgos del Programa y se presentan sus resultados más relevantes. A lo largo del texto, se pone de manifiesto cómo un núcleo esencial del éxito de este tipo de experiencias es la realización de un trabajo sistemático de orientación personal y profesional en todas las fases del proceso.
\end{abstract}

* Inmaculada Egido Gálvez es profesora en la facultad de educación de la UCM. Líneas de investigación: Educación comparada, Política educativa en España, Inclusión de personas con discapacidad intelectual en entornos normalizados.correo-e: miegido@edu.ucm.es

** Rosario Cerrillo Martín es profesora de Facultad de Formación de Profesorado y Educación. Universidad Autónoma de Madrid. Líneas de investigación: Medidas curriculares y organizativas para la atención a la diversidad, Inclusión de personas con discapacidad intelectual en entornos normalizados, Programas de intervención educativa.

*** Asunción Camina Durántez, profesora de la Facultad de Formación de Profesorado y Educación de la Universidad Autónoma de Madrid. Líneas de investigación: Programas de recuperación de las dificultades en el aprendizaje lector, Evaluación criterial de los aprendizajes instrumentales con especial referencia a la lectura, Planes y realidades en la formación de maestros, Inclusión de personas con discapacidad intelectual en entornos normalizados. 
Palabras clave: discapacidad intelectual, orientación profesional, orientación personal, formación laboral, inclusión.

\begin{abstract}
Nowadays, supported employment is one of the most promising strategies in order to achieve the labour inclusion of people with disabilities. This article summarises a research project that includes the implementation and evaluation of a Training Program which is aimed at the job placement of young people with intellectual disability developed in a university context. The text describes the main characteristics of the Program, that it is dedicated to prepare 16 intellectually disabled young to occupy labour positions as secretary's office and office boy. Due to the fact that the training is done in a university environment, the program allows the students to use the premises and the university services to do both the curricular activities and the leisure activities. The main content of the article shows the most relevant achievements of the experience. The results were collected through a systematic evaluation process based in questionnaires applied to the young participants in the Program, to their families and to teachers, in interviews answered by the university students that collaborate in the Program and in the analysis of the diaries of experiences of the disabled young participating in the Program. Besides the increase of their labour expectations and the improvement of their self-esteem as a consequence of their participation in the program, it can be showed how the development of the Program inside of the university gives additional advantages to the students and also to the university community. It is important to highlight that along the text it is expressed that the delivery of personal and career guidance in every stage of the process is one of the keys to the success of this kind of experiences.
\end{abstract}

Key words: intellectual disability, professional guidance, personal guidance, vocational training, inclusion.

\title{
Introducción
}

La igualdad de oportunidades es, sin duda, un principio compartido y una aspiración generalizada en nuestras sociedades. Para las personas con discapacidad, dicho principio se ha traducido en la lucha contra la discriminación y en la búsqueda de la normalización en todas las esferas de su vida. En los años pasados, se produjo una importante evolución en la aplicación de ese principio en entornos como el sanitario o el escolar. En el momento actual, un ámbito clave para la inclusión lo constituye el entorno laboral. La participación de las personas con discapacidad en los procesos económicos y productivos, si bien encuentra todavía muchos obstáculos, es, por tanto, un ámbito prioritario de la acción encaminada a la búsqueda de la igualdad de oportunidades (Rosenqvist, 1990).

Sin embargo, no todos los tipos de discapacidad presentan las mismas dificultades a la hora de conseguir la integración laboral. Mientras las personas con discapacidades motrices o sensoriales pueden, en muchas ocasiones, conseguir y mantener un empleo mediante la realización de adaptaciones del entorno laboral relativamente sencillas, las personas con discapacidad intelectual requieren, en la mayoría de los casos, adaptaciones y cambios más profundos (Alba y Moreno, 2004). Por esta razón, muchas de las experiencias desarrolladas para estas personas en los años pasados han consistido en la creación de centros ocupacionales o entornos especiales de empleo protegido, como los Centros Especiales de Empleo (Fenández e Iglesias, 1999; Ibáñez y Mudarra, 2007). Dichas iniciativas, aunque pueden constituir una opción adecuada en determinados casos, no responden realmente al objetivo de normalización, que requiere la integración de la persona con discapacidad en puestos de trabajo de empresas ordinarias. 
La inclusión social y laboral de las personas con discapacidad...

Una de las estrategias que ha demostrado alcanzar un mayor éxito en la inserción laboral de las personas con discapacidad intelectual en entornos ordinarios de trabajo es el empleo con apoyo, que cuenta ya con una tradición de más de treinta años en otros países (Bayer, Kilsby y Sheam, 2000; Leach, 2002). Aunque en España el empleo con apoyo todavía dista mucho de ser una modalidad de amplio alcance en términos numéricos, lo cierto es que tanto la evolución experimentada durante los últimos años como sus resultados han resultado claramente positivos, por lo que cabe esperar un futuro muy favorable a la creación y consolidación de este tipo de programas (Alomar y Cabré, 2005; Jordán, Verdugo y Vicent, 2005; Villa, 2007). Dicha perspectiva esperanzadora se ha reforzado, además, con la regulación normativa del empleo con apoyo en nuestro país en el año 2007, que puso fin al vacío legal que existía al respecto (Real Decreto 870/2007, de 2 de julio).

El empleo con apoyo puede definirse como la modalidad de empleo de personas con discapacidad encaminado a que éstas puedan acceder, mantenerse y promocionarse en una empresa ordinaria en el mercado de trabajo abierto, con los apoyos profesionales y materiales que sean necesarios, ya sean éstos requeridos de forma puntual o permanente.

Las fases principales del desarrollo de un programa de empleo con apoyo, que incluyen dentro de sí diversas acciones, pueden resumirse en las siguientes (Asociación Española de Empleo con Apoyo, 2008):

- Preparación para la incorporación al mercado laboral: elaboración del perfil profesiográfico del candidato e información y orientación laboral en relación a la búsqueda de trabajo.

- Búsqueda de trabajo activa y personalizada, asegurando un puesto adecuado a cada persona mediante la difusión del programa, la prospección del mercado laboral, el asesoramiento y estimulación a la empresa y el análisis de compatibilidad entre candidato y puesto de trabajo.

- Adaptación y entrenamiento en el puesto de trabajo de la persona con discapacidad.

- Seguimiento del trabajo, con evaluación preventiva de las necesidades del trabajador con discapacidad y de la entidad empleadora, así como detección precoz de situaciones conflictivas y aplicación de medidas correctivas.

Si se considera lo anterior, resulta evidente que el papel del orientador es básico en el desarrollo del empleo con apoyo, tratándose de una figura esencial desde el planteamiento de los programas hasta su finalización. El asesoramiento laboral y la orientación personal y profesional a la persona con discapacidad y, en su caso, a su familia y al entorno laboral que acoge al trabajador son, indudablemente, factores clave para el éxito de esta modalidad de empleo. De hecho, la revisión de buenas prácticas en empleo con apoyo pone de manifiesto que es necesario que se proporcione a la persona con discapacidad una formación previa muy ajustada a los requisitos del perfil laboral y un apoyo individualizado a lo largo de todo el proceso, lo que supone un trabajo muy específico del orientador profesional (Confederación Española de Organizaciones a favor de las Personas con Discapacidad Intelectual, 2000 y Jordán de Urríes, 2003).

En este contexto, tan prometedor como complejo, se sitúa la experiencia llevada a cabo por un equipo de investigación interuniversitario e interdisciplinar para desarrollar y evaluar un Programa de formación para la inserción laboral de jóvenes con discapacidad inte- 
lectual en el ámbito universitario ${ }^{1}$. Dicha experiencia, aunque realizada en un marco contextual concreto (el de la Universidad Autónoma de Madrid) y sujeta a unas características propias, puede ser aplicada, con las modificaciones pertinentes, a otros contextos. En ella, además, se pone de manifiesto cómo un núcleo esencial del desarrollo de este Programa es la realización de un trabajo sistemático de orientación personal y profesional (Izuzquiza y Ruiz, 2005).

El diseño de un Programa de formación para el empleo con apoyo requiere, en primer lugar, conocer los perfiles profesionales demandados por las empresas, lo que implica un proceso sistemático de búsqueda y de contacto con las mismas. En este caso concreto la mayoría de los puestos de trabajo ofertados para los jóvenes con discapacidad intelectual correspondía a las categorías de ordenanza o auxiliar administrativo. Teniendo en cuenta lo anterior, es necesario considerar también los perfiles, capacidades e intereses de los estudiantes que van a tomar parte en el Programa. Sobre la base de ambos tipos de conocimiento es posible detectar las carencias formativas que estos jóvenes tienen para cada puesto de trabajo y proporcionarles una formación previa antes de su incorporación al mundo laboral, que contribuya al éxito de la misma.

Dado el objetivo planteado, la selección adecuada de los jóvenes que toman parte en el Programa es un requisito fundamental, por lo que a todos los candidatos se les ha aplicado un conjunto de instrumentos de evaluación. En concreto, la selección de los estudiantes se ha realizado en función de los resultados obtenidos tras la aplicación de los siguientes de instrumentos de evaluación: WAIS-III, Escala de inteligencia de Wechsler para adultos, K-BIT Test Breve de inteligencia de Kaufman para funciones cognitivas; ICAP Inventario para la Planificación de Servicios y la Programación Individual, para medida de la conducta adaptativa.

Además, como criterios de selección derivados del tipo de trabajo para el que se va a formar a los estudiantes, se encontraba el requisito de que todos los jóvenes que participaran en el Programa poseyeran un amplio grado de autonomía, así como un adecuado conocimiento de la lectura y la escritura.

Finalmente, con estos criterios, el Programa se ha desarrollado, en una primera fase, con un total de 16 jóvenes con discapacidad intelectual con edades comprendidas entre 18 y 29 años. Todas las acciones desarrolladas se orientan a preparar a esos jóvenes en las competencias generales y específicas requeridas para los puestos de trabajo.

Un rasgo destacable y diferencial de esta experiencia es que el Programa de formación se lleva a cabo en un contexto universitario. Los alumnos acuden diariamente a un aula universitaria de la Facultad de Formación de Profesorado y Educación de la Universidad Autónoma de Madrid y están en contacto con otros estudiantes que cursan las Titulacio-

1. El Programa Promentor, financiado por la Fundación Prodis se desarrolla en la Facultad de Formación de Profesorado y Educación de la Universidad Autónoma de Madrid desde el año 2005. Para ello se han firmado tres convenios específicos de colaboración y gestión entre la Fundación General de la UAM y la Fundación Prodis. En este artículo se trata de manera específica el proyecto de investigación titulado "Formación para el empleo e inserción laboral de jóvenes con discapacidad intelectual en el ámbito universitario", que fue financiado en la Convocatoria de Ayudas para la creación o consolidación de grupos de investigación de la Universidad Autónoma de Madrid cofinanciados con la Comunidad de Madrid del curso 2005-2006 (09/SHD/034) y 1levado a cabo por el Grupo de investigación "Inclusión social y laboral de jóvenes con discapacidad intelectual” (Ref. PR-005). 
nes de Magisterio de Educación Especial y de Psicopedagogía, que colaboran voluntariamente en el desarrollo del mismo. El profesorado está formado por profesores universitarios especializados en orientación y discapacidad, junto con profesionales del campo de la Educación Especial. Los alumnos utilizan las instalaciones y servicios de la universidad tanto para el desarrollo del Programa de enseñanza como para las actividades de ocio y tiempo libre.

El diseño del programa de estudios se ha basado en el análisis de las necesidades formativas de los alumnos en relación con los perfiles laborales requeridos por el puesto de trabajo. A partir de ahí se elaboró un plan de estudios de organización modular, de dos años de duración, siendo el primero de ellos de carácter teórico-práctico y el segundo eminentemente práctico. Tras la fase de formación se encuentra la de inserción laboral, que será objeto de un seguimiento exhaustivo durante tres años.

Durante el primer año, el Programa de formación cuenta con las siguientes asignaturas:

- Relaciones interpersonales en la empresa

- Competencias laborales específicas

- Tecnología de la empresa

- Inglés básico

- Comunicación oral y escrita

- Conocimiento lógico-matemático

- Estrategias de pensamiento

- Desarrollo de valores

- Utilización de los servicios de la comunidad

- Habilidades emocionales.

Las materias del segundo año del Programa son:

- Comunicación en la empresa y telecomunicaciones

- Confección de documentos

- Bases de datos y páginas Web

- Archivo de impresos y documentos

- Atención al público

- Comunicación oral y escrita

- Inglés

- Cálculo y contabilidad

- Estrategias de pensamiento

- Habilidades emocionales

- Educación para la salud

- Practicum.

El Practicum se desarrolla durante un semestre completo y es objeto de una planificación detallada en las distintas empresas. Su desarrollo se acompaña de un programa de for- 
mación dirigido al Mediador laboral que apoya al trabajador con discapacidad intelectual en la empresa. No obstante, ya desde el primer año los alumnos participan ocasionalmente en experiencias puntuales de trabajo, con el fin de compaginar las enseñanzas del Programa con su aplicación práctica.

Además de la formación llevada a cabo en el horario lectivo, que ocupa cuatro horas diarias de lunes a viernes, el Programa contempla la realización de un amplio número de actividades culturales, artísticas y deportivas, como visitas a museos y exposiciones, realización de actividades en la naturaleza, asistencia a conciertos, etc. Algunas de esas actividades se diseñan especialmente para los jóvenes participantes en el Programa de formación, mientras otras se realizan dentro de la oferta que la Universidad tiene para el conjunto de los estudiantes.

Junto a lo anterior, las tutorías personales con los estudiantes son una parte fundamental del Programa, puesto que son clave para conseguir una orientación individualizada a cada alumno y para conseguir una comunicación fluida entre los profesores y los estudiantes.

\section{Método}

En paralelo al desarrollo del Programa, se ha llevado a cabo un proceso de evaluación del mismo mediante el diseño de un proyecto sistemático orientado a conocer de la forma más rigurosa posible los resultados obtenidos y a identificar las cuestiones susceptibles de mejora.

La evaluación se ha realizado a partir de las aportaciones de todos los sectores implicados en el mismo: alumnos, profesores, padres y estudiantes colaboradores de la universidad. Los instrumentos de recogida de información fueron diversos, empleándose cuestionarios, entrevistas estructuradas, entrevistas abiertas y diarios de experiencias elaborados por los propios estudiantes.

Los tres cuestionarios dirigidos a los alumnos participantes en el programa, a los padres y madres y a los profesores fueron diseñados ad hoc para este trabajo y siguen las recomendaciones de Babbie (1998) en la redacción de cuestiones y enunciados. El proceso de validación de los mismos se llevó a cabo por medio de expertos y de aplicaciones piloto. Los expertos del equipo de investigación y los responsables del programa llevaron a cabo una revisión de los cuestionarios ítem por ítem y elaboraron dos versiones distintas de cada uno de los instrumentos, que fueron recogidas posteriormente en una tercera versión de síntesis. La aplicación piloto de dicha versión de síntesis se realizó con cinco padres, cinco estudiantes y cuatro profesores y a partir de ella se realizó la redacción definitiva de los cuestionarios.

El objetivo general de los cuestionarios era recoger información relevante sobre la valoración del Programa formativo por parte de los alumnos, los padres y madres, y los profesores. En concreto, la información recopilada a través de los mismos hizo referencia a los siguientes aspectos del Programa:

i. Plan de estudios

ii. Actividades complementarias

iii. Organización y recursos materiales 


\section{iv. Recursos humanos \\ v. Proceso formativo \\ vi. Valoración global del Programa}

Partiendo de esa estructura común, los cuestionarios diferían en el número de preguntas y en la formulación de algunas de ellas en función del colectivo concreto al que se dirigían. Los cuestionarios de estudiantes y de padres y madres plantearon un total de 43 ítems, mientras el de profesores amplió ese número a 65. En todos los casos se utilizó una escala tipo likert con cinco niveles de respuesta (nada adecuado, poco adecuado, indiferente, bastante adecuado y muy adecuado). Además, de dichos ítems, se incluyeron también en cada uno de los cuestionarios tres preguntas abiertas en las que los encuestados podían indicar los aspectos más negativos y más positivos del Programa, así como posibles propuestas de mejora del mismo.

En el caso de los estudiantes colaboradores se optó por la realización de una entrevista semiestructurada, dado el menor conocimiento del Programa por parte de este colectivo. Dicha entrevista versó sobre los mismos seis apartados que los cuestionarios citados.

Por su parte, los diarios personales de experiencias de los jóvenes con discapacidad fueron elegidos como un medio para el conocimiento personal de los perfiles de cada uno, sus relaciones sociales y la satisfacción con el Programa. En ellos, los jóvenes que participan en la experiencia recogen sus impresiones, vivencias e ideas sobre lo que va sucediendo en el transcurso del desarrollo del Programa. Son, por lo tanto, reflexiones subjetivas recogidas con un cierto grado de sistematicidad y continuidad en el tiempo. Nos permiten no sólo conocer los hechos, sino también los sentimientos, ilusiones, expectativas y contradicciones de las personas y son significativos tanto por lo que cuentan como por lo que ocultan.

Dada la naturaleza y las características de la información recogida en los diarios personales, los datos de los mismos han sido analizados con la ayuda del programa informático para análisis de datos cualitativos Atlas.ti 5.2. De acuerdo con los principios de la teoría fundamentada o grounded theory, en esta fase del estudio se trabajó con conceptos, categorías y proposiciones (Pidgeon y Henwood, 1997). Tras la lectura reflexiva de los diarios y el almacenamiento de los datos en el ordenador, se llevó a cabo el proceso de codificación inicial de los mismos, en el que se definieron un total de 36 códigos. Dichos códigos fueron posteriormente agrupados en categorías o familias de códigos relacionados con la valoración del programa, las expectativas de futuro, las relaciones personales establecidas durante la participación en el programa, los sentimientos o las actividades de ocio y tiempo libre. A partir de las relaciones entre los códigos y las familias de códigos se obtuvieron redes conceptuales interrelacionadas entre sí y definidas como "autovaloración", "motivaciones", "experiencias laborales" e "integración laboral" que permitieron contrastar los hallazgos obtenidos con las hipótesis de la investigación y llevar a cabo la interpretación de los resultados.

\section{Resultados}

Los resultados más relevantes del análisis de los datos correspondientes al primer año del desarrollo del Programa son los que se sintetizan a continuación. 


\section{Resultados cuantitativos}

Los datos que se desprenden de la evaluación realizada por parte de los alumnos, los padres y los profesores ponen de manifiesto, en términos generales, una alta valoración del Programa de formación. Los alumnos realizan una valoración global del mismo que se sitúa en un 4,9 en una escala de cinco puntos, mientras los padres le otorgan la máxima puntuación (5) y los profesores la sitúan en un nivel algo inferior, aunque también muy positivo $(4,6)$.

El marco universitario para el desarrollo del Programa es, asimismo, un aspecto valorado muy positivamente por los estudiantes $(4,8)$, los padres $(4,9)$ y los profesores $(5)$.

En relación con los programas de estudio, la valoración general de todas las asignaturas que forman el plan de estudio por parte de los alumnos es buena, ya que la práctica totalidad de los valores se sitúa en un punto superior a 4 en una escala de 1 a 5 . Los resultados más bajos, en opinión de los alumnos, corresponden a la materia de Inglés Básico $(3,8)$, mientras las materias mejor valoradas son Habilidades Emocionales $(4,9)$ y Comunicación Oral y Escrita $(4,8)$. Estas valoraciones coinciden en parte con las que realizan los padres y profesores, aunque estos dos grupos valoran el Inglés Básico de forma más positiva $(4,5)$.

La valoración de las actividades complementarias es, en líneas generales, más baja que la de las asignaturas del plan de estudios, aunque existen algunas diferencias en función de la actividad y del grupo considerado. Así, las visitas a museos son consideradas más positivamente por los profesores y padres $(4,5)$ que por los alumnos (4). Por el contrario, las actividades realizadas en los talleres en entornos naturales son mejor valoradas por los padres $(4,6)$ y los estudiantes $(4,1)$, que por los profesores $(3,3)$.

Las mayores diferencias entre los tres grupos que contestan al cuestionario se producen en relación con las tutorías. En este aspecto, son los padres quienes tienen una opinión más favorable sobre su utilidad $(4,9)$, situándose los estudiantes en una valoración más intermedia $(3,8)$ y siendo los profesores quienes expresan una valoración más baja $(2,8)$. En este caso se pone de manifiesto la diferente percepción que tienen los tres grupos sobre la utilidad de las tutorías, siendo los profesores, como responsables de realizarlas, los que consideran que no se siempre se consiguen en ellas todos los objetivos previstos.

Los resultados sobre las competencias laborales específicas que se pueden adquirir con el Programa formativo y su aplicación al entorno laboral muestran algunas diferencias en función del colectivo analizado. Así, son los padres quienes tienen una visión más optimista de esta cuestión $(4,7)$, seguidos de los alumnos $(4,5)$, mientras los profesores son los que le otorgan una valoración más baja (4). En general, por tanto, todos consideran que el Programa puede contribuir al desarrollo de competencias aplicables en el campo laboral, aunque con ciertos matices, en el caso del profesorado.

En la misma línea, la capacidad del Programa para ayudar a los jóvenes a establecer unas adecuadas relaciones laborales en la empresa es valorada de forma muy positiva por los tres colectivos considerados. En este caso son los profesores quienes otorgan la valoración más alta (5), seguidos de los padres $(4,7)$ y de los estudiantes $(4,4)$. Esta competencia en el ámbito relacional se considera, al menos, tan importante como las competencias específicamente laborales, ya que resulta clave para conseguir una adecuada integración del trabajador discapacitado. 


\section{Resultados cualitativos}

\section{a) Los diarios de experiencias de los jóvenes con discapacidad}

Quizá las aportaciones más ricas las ofrece el análisis de contenido de los diarios de experiencias de los alumnos. En ellos se muestra la satisfacción de estos jóvenes con el Programa de formación, que se refleja en citas como las siguientes: "Estoy orgulloso de estar en la universidad... la idea está estupenda... me encanta este sitio"; "a mí me parece genial este proyecto"; "me siento bien... estoy feliz"; "me han cambiado mucho estos meses en la Facultad... salgo más". Los alumnos reiteran en diferentes ocasiones y de muchas formas en sus diarios la satisfacción con el Programa que están realizando.

De los diarios de los jóvenes se desprende igualmente la satisfacción de los padres y las familias con la experiencia: "me dan la enhorabuena por estar en la universidad... están todos muy contentos, les está gustando mucho"; "mi madre me dice que haga el curso, es bueno para mi"; "es un premio por ser estudioso".

Percibirse con estudiantes universitarios les hace sentirse orgullosos, tanto a ellos como a sus familias. Supone un logro con el que nunca habían soñado. La Universidad, como espacio formativo, representa un universo simbólico capaz de abrirles otros horizontes.

En el ámbito afectivo, son patentes también en los diarios las relaciones positivas entre los compañeros de clase. En ellos afloran sentimientos en numerosas citas, que recogen sus vivencias diarias en el ámbito relacional. Ejemplos de ellas son: "estoy contento por tener compañeros nuevos"; "me lo paso muy bien con la gente de la universidad": "me llevo bien con todos los amigos de la clase". Se trasluce en muchos de los diarios el deseo de ser respetado, comprendido y ayudado por los demás compañeros. En determinados casos, recogen la idea de colaborar y ayudarse mutuamente, aunque también hay casos puntuales en los que se sienten molestos con algunos compañeros e incluso tienden a infravalorar los comportamientos de los otros.

Son también numerosos los fragmentos de los diarios que hacen referencia a sus relaciones con el profesorado. Por lo general, de sus narraciones se desprenden emociones positivas y afectivas hacia el profesorado. Algunos de ellos muestran incluso una valoración extrema: "eres la mejor profesora de la historia del mundo". Sin embargo, en ocasiones expresan también sentimientos negativos hacia algunos profesores, especialmente cuando se les reprende o castiga.

Por lo que se refiere a sus expectativas en lo profesional, en las citas de los diarios se aprecia el valor que otorgan al curso que realizan y el deseo de conseguir una ocupación. Con frecuencia sueñan y se imaginan en sus futuros trabajos. Tienen unas expectativas mucho más altas en este terreno que antes de iniciar el Programa y son conscientes de la necesidad de esforzarse como estudiantes para obtener un empleo.

Las experiencias puntuales de trabajo en empresas que han realizado con el apoyo y la supervisión de los profesores les resultan motivadoras y atractivas, tanto por las actividades que realizan como por las relaciones personales que establecen durante el ejercicio del trabajo. La participación en estas experiencias les estimula y favorece su autoconcepto, su responsabilidad y su dominio emocional, sintiéndose comprometidos con la realización de las tareas. 


\section{b) Entrevistas a los alumnos universitarios que colaboran en el programa}

El análisis de contenido de las entrevistas realizadas a los alumnos universitarios que colaboran en el Programa arroja también algunos resultados interesantes. En ellas se aprecia un cambio de actitudes hacia las personas con discapacidad intelectual, existiendo una mayor apertura y una predisposición más positiva hacia ellos después de haber participado en el Programa. De igual modo, se aprecia un cambio en sus percepciones y una demanda de ampliar el ámbito de relaciones que el grupo de alumnos del Programa mantiene, al considerar que ello contribuiría a eliminar prejuicios y estereotipos sobre la discapacidad intelectual. La convivencia les ha ayudado a comprender que, a pesar de sus limitaciones, estas personas pueden conseguir mucho más de lo que la sociedad espera de ellos. Consideran también que el entorno institucional universitario es el más adecuado para realizar la formación de estos alumnos, aunque discrepan de la ubicación concreta del aula en la que se desarrolla el Programa, por considerarla demasiado aislada del resto, y proponen mejoras en su accesibilidad y ubicación.

\section{Conclusiones}

Aunque es evidente la dificultad que conlleva realizar generalizaciones a partir de los resultados descritos en el apartado anterior, de modo tentativo puede considerarse que el desarrollo de programas específicos de formación para el empleo con apoyo puede ser una vía adecuada para facilitar la integración laboral de las personas con discapacidad intelectual.

En concreto, en la experiencia analizada en este artículo, se aprecia que los alumnos muestran un elevado nivel de implicación en su proceso de formación y que tanto ellos como los padres y profesores consideran que los conocimientos adquiridos mediante el Programa de formación laboral pueden ser de utilidad para el desarrollo de un trabajo en el futuro inmediato. Como consecuencia del desarrollo de este Programa, se produce una mejora de las expectativas personales y profesionales de los estudiantes con discapacidad, así como de su propio autoconcepto, que puede tener una repercusión positiva con vistas no sólo a su integración laboral, sino a su inclusión social, en un sentido más amplio.

El hecho de que un Programa de formación de estas características se desarrolle en un entorno universitario tiene, a su vez, algunas ventajas adicionales. Los padres y alumnos encuentran en el contexto institucional una motivación adicional, mientras los propios estudiantes universitarios cambian sus percepciones y actitudes hacia las personas con discapacidad tras la experiencia de participación con el Programa. Adicionalmente, aunque se trata de un aspecto de difícil constatación, puede esperarse que la propia presencia en la universidad de este tipo de alumnos y la convivencia diaria con ellos en los espacios de ocio y en actividades culturales y deportivas pueda contribuir a un cambio de mentalidad en la propia comunidad universitaria. Este cambio, aunque sea limitado y progresivo, sería un logro fundamental, dado el influjo y la repercusión que la Universidad tiene en nuestras sociedades.

Además de lo anterior, en el Programa desarrollado se ha constatado la importancia de establecer de una forma muy precisa los perfiles profesionales demandados por las empresas a los que las personas con discapacidad intelectual pueden acceder y la necesidad de diseñar la formación orientándola de manera muy específica a las carencias formativas que 
los estudiantes muestran en relación con esos perfiles. Ello requiere un trabajo exhaustivo de contacto con las empresas, así como de selección y de orientación personal y profesional de las personas que participan en el Programa, por lo que resulta vital que los programas de empleo con apoyo cuenten con la presencia de profesionales de la orientación preparados específicamente para esta tarea.

Dados los resultados obtenidos en las experiencias de empleo con apoyo desarrolladas hasta el momento en España y la reciente regulación legal de esta modalidad de trabajo, es previsible que en un futuro próximo se produzca un claro aumento de la demanda de este tipo de profesionales (Benavent, 2003). Las universidades, por tanto, deberían considerar la necesidad de proporcionar esa formación de forma sistemática a los alumnos que cursan estudios relacionados con la orientación. En un momento como el actual, en el que se está produciendo una reforma de la organización y el contenido de los estudios universitarios, la necesidad de contar en el futuro con profesionales bien preparados para diseñar y desarrollar programas de empleo con apoyo debería ser considerada dentro de los perfiles formativos de los egresados de las Facultades españolas. Por una parte, es previsible que exista una demanda creciente de estos profesionales en los próximos años. Por otra, su papel es esencial para contribuir a la integración laboral y, por tanto, social, de las personas con discapacidad.

\section{Referencias bibliográficas}

Alba, A. y Moreno, F. (2004). Discapacidad y Mercado de Trabajo. Madrid: Caja Madrid.

Alomar, E. y Cabré, M. (2005). "El trabajo de jóvenes con discapacidad intelectual en entornos normalizados". Revista Síndrome de Down, 22, 118-124.

Asociación Española de Empleo con Apoyo (2008): El empleo con apoyo. Documento electrónico consultado el 5 de junio de 2008 en http://www.empleoconapoyo.org/aese/article15.html.

Babbie, E. (1998). The Practice of Social Research Belmont. California: Wadsworth. (8th ed.).

Bayer, S. Kilsby, M. y Sheam, J (2000). "The organisation and outcomes of supported employment in Britain". Journal of Vocational Rehabilitation, 12 (2), 137-146.

Benavent, J. A. (2003). "Reflexiones sobre el futuro de la orientación psicopedagógica inmersa en una encrucijada sociocultural". Revista española de orientación y psicopedagogía, 14 (1), 41-59.

Comité Español de Representantes de Personas con Discapacidad (2008). Estrategia global de acción para el empleo de personas con discapacidad 2008-2012. Madrid: Cermi.

Confederación Española de Organizaciones a favor de las Personas con Discapacidad Intelectual (2000). Promoción laboral y empleo. Manuales de buena práctica. Navarra: FEAPS.

Fernández, S. e Iglesias, M. T. (1999). "Análisis de la integración laboral de las personas con discapacidad. Una aplicación en el municipio de Gijón (Asturias)". Revista Española de Orientación y Psicopedagogía, 10 (18), 331-351.

Ibáñez, P. y Mudarra, M. J. (2007). Integración sociolaboral: Trabajadores con discapacidad en Centros Especiales de Empleo. Madrid: Dykinson.

Izuzquiza, D. y Ruiz, R. (2005). "Formación para la inserción laboral. Un proyecto conjunto entre la Facultad de Formación de Profesorado y Educación de la Universidad Autónoma de Madrid y la Fundación Prodis". Revista Síndrome de Down, 22, 125-131.

Jordán de Urríes, F. B. (2003). "Empleo con apoyo: Buenas prácticas". Revista de la Federación Española del Síndrome de Down, 24, 18-21. 
Jordán de Urríes, F. B.; Verdugo, M. A. y Vicent, C. (2005). Análisis de la evolución del empleo con apoyo en España. Madrid: Real Patronato sobre Discapacidad.

Leach, S. (2002). "Empleo con apoyo: buenas prácticas basadas en principios claros". En M. A. Verdugo y F. B. Jordán, B. (coords.). Hacia la integración plena mediante el empleo. Actas VI Simposio Internacional de Empleo con Apoyo. Salamanca: INICO.

Pidgeon, N. y Henwood, K. (1997). "Grounded theory: practical implementation”. En J. Richardson (ed.): Handbook of qualitative research methods for Psycology and the social sciences. Leicester: BPS Books.

Real Decreto 870/2007, de 2 de julio, por el que se regula el programa de empleo con apoyo como medida de fomento de empleo de personas con discapacidad en el mercado ordinario de trabajo (BOE 14 de julio de 2007).

Rosenqvist, J. (1990). "Mental retardation and work opportunities on teachers', managers' and supervisors' conceptions of the students' possibilities in their working life". European Journal of Special Needs Education, (5) 1, 31-42.

Villa, N. (2007). La inclusión progresiva de las personas con discapacidad intelectual en el mundo laboral. Madrid: Ministerio de Trabajo y Asuntos Sociales.

Fecha de recepción: 09-12-2007

Fecha de revisión: 10-03-2009

Fecha de aceptación: 05-05-2009 